\title{
The physics of top, $W$ and $Z$ from LHC, Tevatron and HERA.
}

\author{
Elizaveta Shabalina*t \\ II. Physikalisches Institut, Universität Göttingen \\ E-mail: Elizaveta.Shabalina@cern.ch
}

We summarize recent experimental results in electroweak and top quark physics presented at the conference. This overview covers new measurements of the properties of top quark and $W$ and $Z$ bosons from the LHC, Tevatron and HERA.

35th International Conference of High Energy Physics - ICHEP2010,

July 22-28, 2010

Paris France

* Speaker.

$\dagger$ The author would like to thank D. Denisov, T. Schwarz, F. Margaroli, C. Schwanenberger, F. Deliot, J. Stark, L. Nodulman, M. Lancaster, K. Krüger, A. Heinson, R. Hawkings, W. Verkerke, F.-P. Schilling, M. Verzocchi, G. Bernardi, M. Grünwald and all speakers of track 02 session for their contributions to the talk. 


\section{Introduction}

The standard model (SM) of particle physics has been extremely successful in describing electroweak (EW) phenomena and properties of the top quark. The Large Hadron collider (LHC) at CERN that started its operation in March 2010 provides a new energy frontier to perform precision measurements in top and EW sectors which compliment direct searches for new physics and allow to explore higher mass scales through virtual effects. Due to their large production rates, $W$ and $Z$ bosons and top quarks represent important backgrounds to Higgs boson and new physics searches and have to be well understood.

\section{2. $W$ and $Z$ boson production}

Measurements of the inclusive and differential production cross sections of the $W$ and $Z$ bosons at hadron colliders allow to test several aspects of the SM simultaneously, including the perturbative QCD calculations and the parton distribution functions (PDF).

\section{1 $W$ and $Z$ bosons at the $\mathrm{LHC}$}

The CMS and ATLAS experiments presented first measurements of the $W \rightarrow \ell v$ and $Z / \gamma^{*} \rightarrow$ $\ell \ell(\ell=e, \mu)$ production cross sections in proton-proton collisions at $\sqrt{s}=7 \mathrm{TeV}$ using data set of up to $225 \mathrm{nb}^{-1}$. With the integrated luminosity of $198 \mathrm{nb}^{-1}$ CMS collaboration measures $\sigma(p p \rightarrow W+X \rightarrow \ell v+X)=9.22 \pm 0.24$ (stat) \pm 0.47 (syst) \pm 1.01 (lumi) nb and $\sigma(p p \rightarrow$ $\left.Z / \gamma^{*}+X \rightarrow \ell^{+} \ell^{-}+X\right)=0.882_{-0.073}^{+0.077}(\text { stat })_{-0.036}^{+0.042}($ syst $) \pm 0.097$ (lumi) nb for the dilepton invariant mass range [60,120] GeV [1], in agreement with the theoretical next-to-next-to-leading order (NNLO) predictions of $10.44 \pm 0.52 \mathrm{nb}$ and $0.97 \pm 0.04 \mathrm{nb}$ [2], respectively. More recent measurements by the ATLAS collaboration with integrated luminosity of approximately $320 \mathrm{nb}^{-1}$ yield $\sigma(p p \rightarrow W+X \rightarrow \ell v+X)=9.96 \pm 0.23$ (stat) \pm 0.50 (syst) \pm 1.1 (lumi) nb and $\sigma(p p \rightarrow$ $\left.Z / \gamma^{*}+X \rightarrow \ell^{+} \ell^{-}+X\right)=0.82 \pm 0.06$ (stat) \pm 0.05 (syst) \pm 0.09 (lumi) nb within the invariant mass window $[66,116] \mathrm{GeV}$. Figure 1 shows $W$ boson transverse mass distributions in the muon channel from CMS and in the electron and muon channels from ATLAS in data compared to the signal and background models obtained from the Monte Carlo (MC) simulation. Both experiments also measured production cross sections for $W^{+}$and $W^{-}$separately and $W / Z$ and $W^{+} / W^{-}$cross section ratios. The ratios are precisely predicted by NNLO theoretical calculations but do not suffer from experimental uncertainty from integrated luminosity, which cancels, along with other uncertainties, which either fully or partially cancel. The $W^{+} / W^{-}$cross section ratio measurement is of special interest at the LHC since, in contrast to $p \bar{p}$ collisions, the cross sections for $W^{+}$and $W^{-}$ production in $p p$ interactions are expected to be different due to different distributions of $u$ and $d$ valence quarks. All presented measurements agree between channels and experiments, and no disagreement with the SM calculations are found within rather large experimental uncertainties.

\section{2 $W$ bosons at the HERA}

The signature of $W$ boson production at ep collisions at HERA includes high $p_{T}$ lepton, missing transverse energy from a neutrino and a hadronic system with transverse momentum $p_{T}^{X}$. The $\mathrm{H} 1$ and ZEUS experiments combined their measurements of the $W$ production cross section as a 



Figure 1: $W$ boson transverse mass distributions in the muon channel from CMS (left plot) and in the electron (middle) and muon (right) channel from ATLAS in data compared to the signal and background models from MC simulation.

function of $p_{T}^{X}$ in a data set of $0.98 \mathrm{fb}^{-1}$ [4]. The result is consistent with the SM prediction. Excess of data over SM expectation at $p_{T}^{X}>25 \mathrm{GeV}$ present in the earlier $\mathrm{H} 1$ data is not observed in the combined measurement.

\section{3 $\mathrm{Z} / \gamma^{*}$ differential distributions at the Tevatron}

Large sample of $Z / \gamma^{*} \rightarrow \ell^{+} \ell^{-}$events collected at the Fermilab Tevatron collider in $p \bar{p}$ collisions at $\sqrt{s}=1.97 \mathrm{TeV}$ provides an excellent ground for testing the QCD due to small experimental backgrounds and the absence of color flow between the initial and final states. As any momentum of the $Z / \gamma^{*}$ in the plane transverse to the incoming beams has to be balanced by a recoiling system resulting mainly from the radiation in the initial state, the $Z / \gamma^{*}$ transverse momentum $\left(p_{T}\right)$ distribution is sensitive to the nature of such radiation and is widely used as a probe for the underlying process. The D0 collaboration presented a new measurement of the $Z / \gamma^{*} \rightarrow \mu^{+} \mu^{-} p_{T}$ distribution in the range $0-330 \mathrm{GeV}$ using $0.97 \mathrm{fb}^{-1}$ data set [5]. The result is presented at the level of particles entering the detector and is compared to the predictions of fixed order and resummation calculations [6], parton shower generators PYTHIA [7] and HERWIG [8] and matrix element and parton shower generators ALPGEN [9] and SHERPA [10] which show variable agreement with the data. This measurement provides an important input for the tuning of theoretical predictions to better describe hadron collider data. However, in the region of low $Z / \gamma^{*} p_{T}$, it is dominated by uncertainties on the correction for experimental resolution and efficiency. Using a data set of $7.3 \mathrm{fb}^{-1}$ the D0 collaboration studied the distribution of the variable $\phi_{\eta}^{*}[11]$, defined as $\phi_{\eta}^{*}=\tan \left(\phi_{\text {acop }} / 2\right) \sin \left(\theta_{\eta}^{*}\right)$, where $\phi_{\text {acop }}$ is the acoplanarity angle, given by $\phi_{\text {acop }}=\pi-\Delta \phi^{\ell \ell}$, and $\Delta \phi^{\ell \ell}$ is the difference in azimuthal angle, $\phi$, between the two lepton candidates. The variable $\theta_{\eta}^{*}$ is a measure of the scattering angle of the leptons with respect to the proton beam direction in the rest frame of the dilepton system. The variable $\phi_{\eta}^{*}$ probes the same physical effects as $Z / \gamma^{*} p_{T}$ but is less sensitive to the effects of experimental resolution since it depends exclusively on the directions of the two leptons, which are measured with much higher precision than the momenta of the leptons. Figure 2 (left) shows the ratio of the $\phi_{\eta}^{*}$ distributions in data in electron and muon channels to the predictions of RESBOS NLO generator [6]. While the general shape of $\phi_{\eta}^{*}$ distribution is well described by REsBos over the full range of the $\phi_{\eta}^{*}$ variable, the width of the $\phi_{\eta}^{*}$ distribution in data becomes narrower with 
increasing $|y|$ faster in the data than is predicted by REsBos. This is the opposite of the behavior expected from the small- $x$ broadening hypothesis [12].

The presence of both vector and axial-vector couplings of the $Z$ bosons to fermions in $q \bar{q} \rightarrow$ $Z / \gamma^{*} \rightarrow \ell^{+} \ell^{-}$gives rise to an asymmetry in the polar angle $(\theta)$ of the negatively charged lepton momentum relative to the incoming quark momentum in the rest frame of the lepton pair. The angular differential cross section can be written as $d \sigma / d \cos \theta=A\left(1+\cos ^{2} \theta\right)+B \cos \theta$, where $\mathrm{A}$ and $\mathrm{B}$ depend on the weak mixing angle $\theta_{W}$, and the forward-backward charge asymmetry, $A_{F B}$, is determined by $\cos \theta$ term. Experimentally, it is measured as a difference in the number of the forward $(\cos \theta>0)$ and backward $(\cos \theta<0)$ events normalized to the total number of events. New measurement of $A_{F B}$ as a function of dielectron mass in the range $50<M_{e e}<600 \mathrm{GeV}$ by the CDF collaboration using $4.1 \mathrm{fb}^{-1}$ of data shows good agreement with PYTHIA prediction [13]. The earlier study by the D0 collaboration [14] with $1.1 \mathrm{fb}^{-1}$ data set used $A_{F B}$ to extract the effective weak mixing angle $\sin ^{2} \theta_{\text {eff }}^{W}=0.2326 \pm 0.0018$ (stat) \pm 0.0006 (syst). With more than $8 \mathrm{fb}^{-1}$ of data recorded by the Tevatron experiments by now, a combined measurement of $A_{F B}$ by the CDF and D0 collaborations using electron and muon final states could lead to a measurement of $\sin ^{2} \theta_{\text {eff }}^{W}$ with a precision comparable to that of the current world average.

\section{Diboson physics}

Precision measurements of diboson processes are of interest for several reasons. Diboson production constitutes a very important background to Higgs and SUSY searches, and its precise knowledge and accurate modeling is necessary to observe these small signals.

The SM predicts non-zero tree-level couplings among various gauge bosons through triple gauge couplings (TGC). General lagrangians for charged $(W W \gamma / W W Z)$ and neutral $(Z Z \gamma / Z \gamma \gamma)$ interactions have 14 and 8 TGC parameters, respectively. Assuming electromagnetic gauge invariance and CP conservation the number of parameters is reduced to five for the former. In the SM, three of them, $g_{1}^{Z}$ and $\kappa_{V}$, where $V=Z$ or $\gamma$, are expected to be unity, and the rest, $\lambda_{V}$, to be zero. CP conservation allows for four neutral TGC parameters, $h_{i}^{V}$, where $i=3,4$, all of which are predicted to be zero in the SM. In the presence of new physics TGCs can deviate from the SM predictions, and thus serve as a probe for physics beyond the SM. All diboson signals have been observed at the Fermilab Tevatron collider by the CDF and D0 experiments in recent years. New measurements of the cross sections and couplings were shown for $Z \gamma, W Z$ and $Z Z$ production.

The CDF collaboration presented a new measurement of $Z \gamma$ production using $Z$ boson decays into electron, muon and neutrino pairs with $5.1 \mathrm{fb}^{-1}$ data set [15]. Photon $E_{T}$ spectrum shown in Fig. 2 (middle) was used to set limits on the $h_{i}^{V}$ couplings. The measurement of $W Z$ production cross section in the three-lepton and missing transverse energy final state was updated using $\sim 6$ $\mathrm{fb}^{-1}$ of integrated luminosity and two different techniques. The first one uses NeuroBayes neural network to distinguish $W Z$ signal from backgrounds [16], and the second one measures the ratio $\sigma(p \bar{p} \rightarrow W Z) / \sigma(p \bar{p} \rightarrow Z)$ [17]. The latter approach allows to reduce systematic uncertainties. Both results are consistent with SM. Studying the same decay mode with $4.1 \mathrm{fb}^{-1}$ of data the D0 collaboration sets limits on the coupling parameters $g_{1}^{Z}, \lambda_{Z}$ and $\kappa_{Z}[18]$ based on $Z p_{T}$ distribution shown in Fig. 2 (right). All TGCs measured so far agree with SM within large uncertainties. A 


\begin{tabular}{l|c|c||c|c||c}
\hline \hline process & \multicolumn{2}{|c||}{$\mathrm{D} 0$} & \multicolumn{2}{c||}{ CDF } & theory \\
\hline & $\sigma[\mathrm{pb}]$ & $\mathscr{L}\left[\mathrm{fb}^{-1}\right]$ & $\sigma[\mathrm{pb}]$ & $\mathscr{L}\left[\mathrm{fb}^{-1}\right]$ & $\sigma[\mathrm{pb}]$ \\
\hline$W W$ & $11.5 \pm 2.2$ & 1.1 & $12.1_{-1.7}^{+1.8}$ & 3.6 & $12.0 \pm 0.7$ \\
$W Z$ & $3.9_{-0.9}^{+1.1}$ & 4.1 & $4.1 \pm 0.7$ & 6.0 & $3.46 \pm 0.21$ \\
$Z Z$ & $1.60 \pm 0.65$ & 2.7 & $1.7_{-0.7}^{+1.2}$ & 6.0 & $1.4 \pm 0.1$ \\
\hline \hline
\end{tabular}

Table 1: Summary of the measured diboson cross sections at the Tevatron, with the corresponding integrated luminosity, and the theoretical predictions.

summary of the measured diboson production cross sections and theoretical calculations is given in Table 1.


Figure 2: Left: Ratio of the $\phi_{\eta}^{*}$ distributions in data to the predictions of RESBOS in different $Z / \gamma^{*}$ rapidity $y$ regions. The yellow band around RESBos prediction represents uncertainty due to PDF and the QCD scale. RESBOs predictions are shown for different choices of parameters. Middle: Expected and measured photon $E_{T}$ distributions for SM (black) and for anomalous couplings (red and blue). Right: The $Z$ boson $p_{T}$ spectrum from data (points), total background (dark histogram), the sum of SM WZ signal and background (open histogram), and two anomalous coupling models (dashed and dotted histograms) for D0 analysis.

\section{4. $W$ mass and width}

The precise measurement of masses and widths of fundamental particles provides important information on the internal consistency of the SM. These measurements are sensitive to the effects of virtual heavy particles in loops, and thus provide information about higher mass scales. The $W$ boson mass is the most important example, with a quadratic dependence on the top quark mass and a logarithmic dependence on the Higgs boson mass through radiative corrections. The most precise single measurement was performed by D0 using $1 \mathrm{fb}^{-1}$ of integrated luminosity and yields $m_{W}=80.401 \pm 0.043 \mathrm{GeV}$ [19]. Combination with the measurements by the CDF, LEP and SLD measurements resulted in a new world average $W$ boson mass of $m_{W}=80.339 \pm 0.031 \mathrm{GeV}$ [20].

Using the same technique as for the $W$ mass measurement and the same data set the D0 collaboration extracted the $W$ boson width from the shape of the transverse mass distribution [21]. The result, $\Gamma_{W}=2.028 \pm 0.072 \mathrm{GeV}$, is in agreement with the SM prediction. It was recently combined with the earlier measurements from other experiments yielding $\Gamma_{W}=2.085 \pm 0.042 \mathrm{GeV}$ [20]. 


\section{Top quark production}

The large sample of top-antitop quark pairs collected by the D0 and CDF experiments at the Fermilab Tevatron collider allows to perform precision measurements of the fundamental top quark characteristics, such as its production cross section and mass, study a broad variety of top quark production and decay properties to address the question whether the top quark is indeed the particle predicted by the SM.

The most precise measurements of the $t \bar{t}$ cross section have been achieved so far in the $\ell+$ jets channel, where one of $W$ bosons from $t \rightarrow W b$ SM decay decays leptonically and the other one hadronically. This channel has a good rate and manageable background dominated by the production of $W$ bosons in association with heavy and light flavor jets $(W+$ jets). To discriminate signal from background two approaches are used. The first approach makes use of the distinct kinematic features of a $t \bar{t}$ event arising from its large mass. The second approach requires that at least one of the jets per event is identified as a $b$-jet. Recent cross section measurements by the CDF and D0 collaborations [22] are limited by systematic uncertainties, the largest uncertainty coming from the luminosity measurement. By measuring the ratio of the $t \bar{t}$ to $Z$ boson cross section and, thus, replacing the luminosity uncertainty with the smaller theoretical and experimental uncertainties on $Z$ cross section, $\mathrm{CDF}$ achieved the precision of $7 \%$ by combining the results from two methods and measured $\sigma_{t \bar{t}}=7.70 \pm 0.52 \mathrm{pb}$ in $4.6 \mathrm{fb}^{-1}$ data set for top quark mass $m_{t}=172.5 \mathrm{GeV}$.

The CDF collaboration presented the first search for boosted top quarks, when all top quark decay products are contained in one jet, using $5.95 \mathrm{fb}^{-1}$ of data [23]. This study is of special importance for the LHC which discovery potential in the top quark sector depends significantly on the ability to reconstruct such objects. CDF found 103 candidate events in a sample selected by requiring either two massive jets or one massive jet and high missing transverse energy on a background of $76 \pm 10$ (stat) ${ }_{-20}^{+26}$ (syst) events and set upper limit on the SM production cross section of $t \bar{t}$ events with at least one top quark with $p_{T}>400 \mathrm{GeV}$ of $54 \mathrm{fb}$ at $95 \%$ C.L.

Electroweak production of the single top quarks was observed by the CDF and D0 collaborations in 2009 [24]. It allows to directly probe Wtb interaction. Following the observation of the combined $s$ - and $t$-channel production, the D0 collaboration published $4.8 \sigma$ evidence for the $t$-channel production [25]. Combination of the CDF and D0 results for the combined $s$ - and $t$ channel production yields $\sigma=2.76_{-0.47}^{+0.58} \mathrm{pb}$ which translates into a direct measurement of CKM matrix element $\left|V_{t b}\right|=0.88 \pm 0.07$ with a 95\% C.L. lower limit of $\left|V_{t b}\right|>0.77$.

\section{Top quark properties}

Properties of the top quark, such as top quark charge, lifetime, production mechanism, branching fractions and couplings, are well defined in the SM, and their measurements provide a probe of its validity. The top quark mass is a free parameter of the SM. Its surprisingly large value suggests a unique role of the top quark in the mechanism of EW symmetry breaking. Together with the $W$ boson mass, it provides an important indirect constraint on the mass of the SM Higgs boson. The most recent measurement from the CDF experiment using $5.6 \mathrm{fb}^{-1}$ of data in $\ell+$ jets channel [26] yields $m_{t}=173.0 \pm 0.7$ (stat) \pm 0.6 (JES) \pm 0.9 (syst) GeV, corresponding to a total uncertainty of $1.2 \mathrm{GeV}$ and a relative uncertainty of $0.7 \%$. Its combination with the other measurements per- 
formed in different channels by the CDF and D0 collaborations results in the Tevatron average top quark mass of $m_{t}=173.3 \pm 1.1 \mathrm{GeV}$ [27]. The largest uncertainty on the combined mass of 0.46 $\mathrm{GeV}$ comes from the statistical component of the jet energy calibration determined from the fit to data followed by the uncertainties associated with the different aspects of the signal modeling.

Top quark mass measurements assume that the top quark mass is equal to the antitop quark mass as demanded by CPT theorem. The CDF collaboration dropped this assumption and studied the top-antitop quark mass difference in $5.6 \mathrm{fb}^{-1}$ of data [28]. CDF found $\Delta M=-3.3 \pm 1.4($ stat $) \pm$ 1.0 (syst) $\mathrm{GeV}$, in agreement with SM within large uncertainties dominated by statistical one.

The lifetime of the top quark is a fundamental property that has not been measured precisely so far because it is extremely short and, thus, unlike other heavy quarks, the top quark does not form long-lived hadrons that can be observed in the detector. CDF performed a direct measurement of the top quark width in the $\ell+$ jets channel using $4.3 \mathrm{fb}^{-1}$ of data by studying the reconstructed top quark mass spectrum [29] which is sensitive to the width $\Gamma_{t}$. However, since the experimental resolution is much smaller than $\Gamma_{t}$ the analysis sets only an upper limit $\Gamma_{t}<7.5 \mathrm{GeV}$ at 95\% C.L. D0 measured $\Gamma_{t}$ indirectly from the $t$-channel single top quark production cross section proportional to the partial width $\Gamma(t \rightarrow W b)$. This method assumes that couplings in the top quark production and decay are the same. The total width is found to be $\Gamma_{t}=1.99_{-0.55}^{+0.69} \mathrm{GeV}$ for $m_{t}=170 \mathrm{GeV}$ corresponding to the lifetime of $\tau=\left(3.3_{-0.9}^{+1.3}\right) \times 10^{-25} \mathrm{~s}$ [30], in agreement with SM.

Extremely short lifetime of the top quark allows to study its spin at production since hadronization effects do not deteriorate spin information, and the latter is reflected in the angular distributions of the top quark decay products. The CDF and D0 collaborations have measured the spin correlations between $t$ and $\bar{t}$ in the dilepton channel by analyzing the double differential angular distributions of leptons (D0) and leptons and $b$ and $\bar{b}$ quarks (CDF) using data with an integrated luminosity of up to $4.2 \mathrm{fb}^{-1}$ (D0) [31] and $2.8 \mathrm{fb}^{-1}$ (CDF) [32], respectively. The most recent measurement was performed by the CDF collaboration in the $\ell+$ jets channel with a data set of $5.3 \mathrm{fb}^{-1}$ [33]. Spin correlation parameter $\kappa$ in the beam basis was found to be $\kappa_{\text {beam }}=0.72 \pm 0.64$ (stat) \pm 0.26 (syst). All measurements of the spin correlations performed at the Tevatron so far are consistent with SM but their sensitivity is significantly statistically limited.

Measurements of the charge asymmetry in top quark production, which can be observed at the Tevatron as a forward-backward asymmetry, sparked a large interest among theorists because previous inclusive measurements by the CDF and D0 collaborations had found large positive asymmetries that were nevertheless consistent with the NLO predictions within large uncertainties. The deviation of the observed forward-backward asymmetry from the SM prediction can indicate the contribution from the unexpected new $t \bar{t}$ production channels. New CDF analysis uses $5.6 \mathrm{fb}^{-1}$ data set [34] and determines the asymmetry at the parton level to be $A_{f b}=$ $0.150 \pm 0.050$ (stat) \pm 0.024 (syst) in the laboratory frame, in agreement with the previous measurements. The study of $A_{f b}$ as a function of the rapidity difference $\Delta y=y_{l e p}-y_{\text {had }}$ between top quarks decaying leptonically and hadronically in two regions of the $t \bar{t}$ rapidity difference yields $A_{f b}(|\Delta y|<$ $1.0)=0.026 \pm 0.104$ (stat) \pm 0.055 (syst) and $A_{f b}(|\Delta y|>1.0)=0.611 \pm 0.210$ (stat) \pm 0.141 (syst), to be compared to the MCFM model predictions $0.039 \pm 0.006$ and $0.123 \pm 0.018$ for the inner and outer rapidities, respectively. The updated measurement from the D0 collaboration with $4.3 \mathrm{fb}^{-1}$ data set finds reconstructed $A_{f b}=(8 \pm 4) \%$ [35], while MC@NLO prediction is $1_{-1}^{+2} \%$. Figure 3 (left) presents the distribution of the reconstructed rapidity of the top quark $y_{t}$ in data compared to 
the sum of signal and background in the study by CDF. The legend shows the reconstructed $A_{f b}$. Middle plot shows the asymmetry in the $t \bar{t}$ rest frame at small and large $|\Delta y|$, including correction to the parton level and comparison with the MCFM prediction. Right plot compares reconstructed $\Delta y$ in data with the signal and background model for the D0 analysis. Distributions presented in the right and left plots of Fig. 3 show the difference in shapes between the asymmetry predicted by the simulation and the observed one in data.
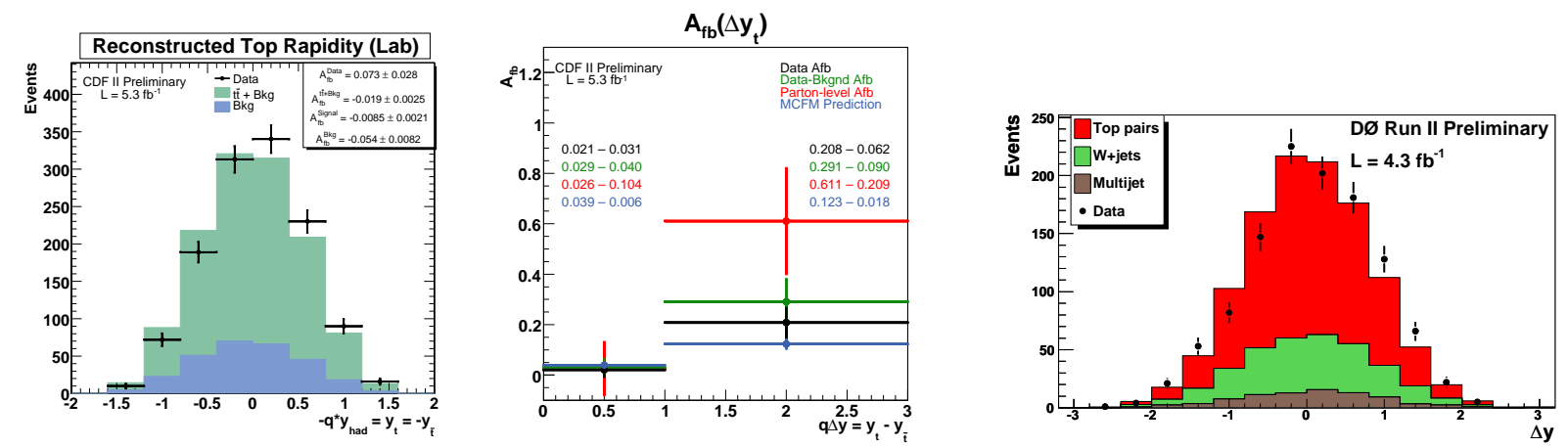

Figure 3: Left: distribution of the reconstructed rapidity of the top quark $y_{t}$ in data compared to the sum of signal and background. Center: the asymmetry in the $t \bar{t}$ rest frame at small and large $|\Delta y|$. Right: reconstructed $\Delta y$ in data compared to the signal and background model.

\section{Electroweak fit}

The measurements of EW observables, such as cross sections, masses and various couplings of the heavy EW gauge bosons confront the theory in the global fits performed by the LEP EW Working group [20]. The LEPEWWG fits assume the SM with one Higgs boson. The inputs include five $Z$ line shape and forward-backward asymmetries, two polarized leptonic asymmetries, one hadronic charge asymmetry, six heavy quark flavor results, the top quark and $W$ boson masses, and $W$ width. The current constraint from the fit on the Higgs boson mass is presented in Fig. 4. where the solid blue line ellipse shows the direct $W$ and top quark mass measurements, the region marked by a dashed red line shows the indirect determinations, and the lines show the SM prediction for particular Higgs masses. The white area inside the green region corresponds to the Higgs masses $158<m_{H}<175 \mathrm{GeV}$ excluded by the Tevatron experiments [36]. Higgs boson mass is predicted to be $m_{H}=89_{-26}^{+35} \mathrm{GeV}$ at $68 \%$ C.L. (not including theory uncertainties) with the upper limit of $m_{H}<158 \mathrm{GeV}\left(m_{H}<185 \mathrm{GeV}\right)$ at $95 \%$ C.L. if direct limit of $114 \mathrm{GeV}$ from LEP is excluded (included) in the fit. The updated experimental inputs to the latest fit, the Tevatron average top quark mass and the world average $W$ boson width, had a small effect on $m_{H}$ compared to the previous fit. It is clear from Fig. 4 that a significant improvement of the uncertainty on the $W$ mass is critical for further tightening the limits on $m_{H}$. 

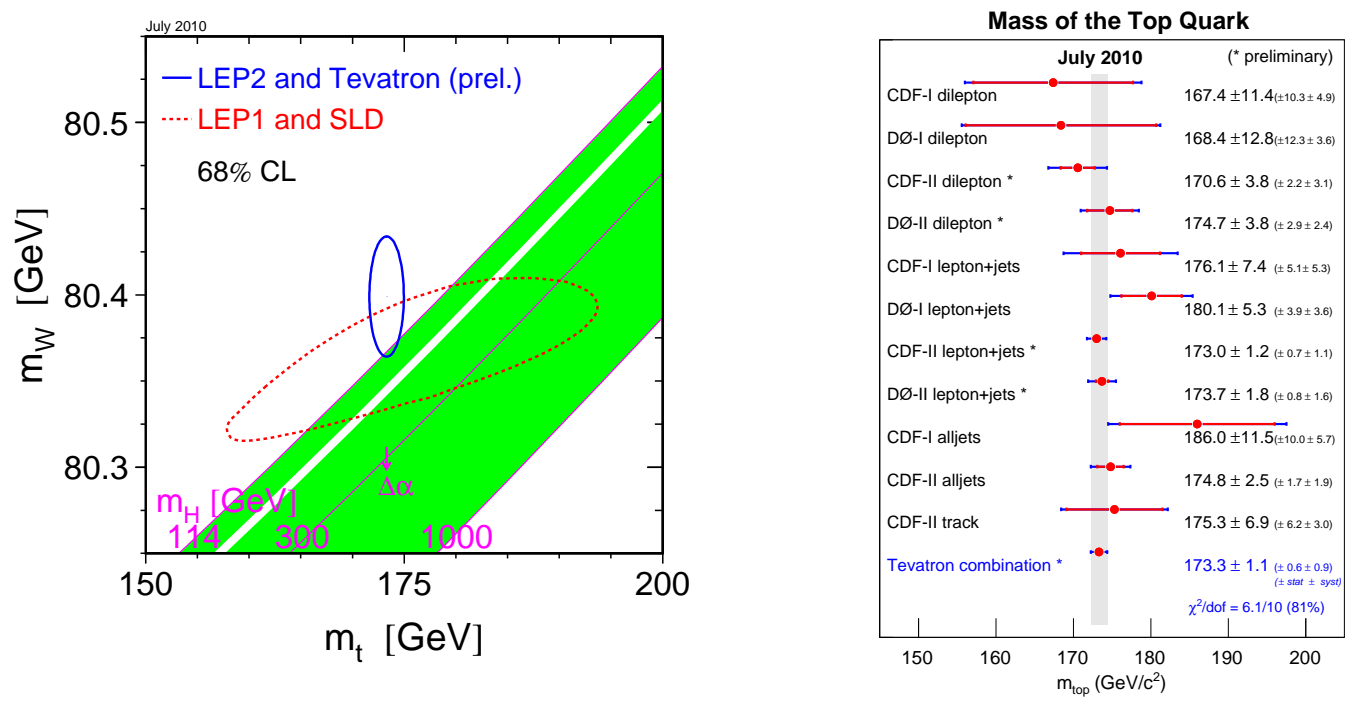

Figure 4: Left: the indirect constraints from the EW fits (dashed red curve) and the direct measurements (solid blue curve) of the top quark and $W$ boson masses. Diagonal lines show SM predictions for different values of the Higgs mass. Right: summary of the top quark mass measurements at the Tevatron and their combination.

\section{Conclusions and outlook}

The measurements in EW and top quark sectors at the Tevatron have reached unprecedented precision. The uncertainty on the top quark pair production cross section challenges the accuracy of the theoretical calculations and, along with the uncertainty on the top quark mass, exceeds the Tevatron goal. Significant improvement of uncertainty on the $W$ mass is desirable to better constrain Higgs boson mass from the EW fit. Tevatron experiments have achieved impressive sensitivity in studies of the top quark properties and explored such new ones as top quark width and spin correlations.

Measurements of EW gauge boson differential distributions provide valuable information for the tuning of theoretical models to better describe hadron collider data. Diboson signatures are well established and used to study couplings and possible signals of new physics. No significant deviations from the SM have been observed in electroweak and top quark sectors so far.

First EW gauge boson measurements from the LHC demonstrate excellent performance of the CMS and ATLAS detectors and their readiness for exciting $W, Z$ and top quark physics with a larger data set expected in 2011.

\section{References}

[1] CMS Collaboration, CMS-PAS-EWK-10-002.

[2] K. Melnikov and F. Petriello, Phys.Rev. D74 114017 (2006) [hep-ph/ 0609070 ]; K. Melnikov and F. Petriello, Phys.Rev.Lett. 96231803 (2006) [hep-ph/ 0603182$].$

[3] ATLAS Collaboration, arXiv:1010.2130v1 [hep-ex]. 
[4] H1 and ZEUS Collaborations, JHEP 03035 (2010) [arXiv: $0911.0858 v 1$ [hep-ex] ].

[5] D0 Collaboration, Phys.Lett. B693 522 (2010) [arXiv:1006.0618v3 [hep-ex]].

[6] C. Balazs, C. P. Yuan, Phys.Rev. D56 5558 (1997) [hep-ph/9704258].

[7] T. Sj̈̈trand, S. Mrenna and P. Skands, JHEP 05026 (2006).

[8] G. Corcella et. al., JHEP 01010 (2001).

[9] M. L. Mangano et al., JHEP 07001 (2003).

[10] T. Gleisberg et al., JHEP 02007 (2009).

[11] D0 Collaboration, arXiv:1010.0262v2 [hep-ex].

[12] P. Nadolsky, D. R. Stump, C. P. Yuan, Phys.Rev. D64 114011 (2001) [hep-ph/ 0012261 ]; S. Berge, P. Nadolsky, F. Olness, C. P. Yuan, Phys.Rev. D72 033015 (2005) [hep-ph/ 0410375$].$

[13] CDF Collaboration, CDF public note 10312.

[14] D0 Collaboration, Phys.Rev.Lett. 101191801 (2008) [arXiv.org:0804 . 3220].

[15] CDF Collaboration, http://www-cdf.fnal.gov/physics/ewk/2010/Z_gamma/index.html.

[16] CDF Collaboration, CDF public note 10176.

[17] CDF Collaboration, CDF public note 10238.

[18] D0 Collaboration, arXiv:1006.0761 [hep-ex].

[19] D0 Collaboration, Phys.Rev.Lett. 103141801 (2009) [arXiv:0908.0766v2 [hep-ex]].

[20] LEP Electroweak Working Group, http://lepewwg.web.cern.ch/LEPEWWG/plots/summer2010/.

[21] D0 Collaboration, Phys.Rev.Lett. 103231802 (2009) [arXiv:0909.4814v2 [hep-ex] ].

[22] D0 Collaboration, D0 public note 6037-CONF; CDF collaboration, Phys.Rev.Lett. 105012001 (2010) [arXiv:1004.3224v2 [hep-ex]]; CDF Collaboration, CDF public note 10137.

[23] CDF public note 10234.

[24] D0 collaboration, Phys.Rev.Lett. 103092001 (2009) [arXiv:/0903.0850 [hep-ex] ]; CDF collaboration, Phys.Rev.Lett. 103092002 (2009) [arXiv:/0903.0885 [hep-ex] ].

[25] D0 collaboration, Phys.Lett. B683 363 (2010) [arXiv:0907.4259 [hep-ex] ].

[26] CDF Collaboration, CDF public note 10191.

[27] The Tevatron Electroweak Working group, arXiv:1007.3178v1 [hep-ex].

[28] CDF Collaboration, CDF public note 10173.

[29] CDF Collaboration, CDF public note 10035.

[30] D0 collaboration, arXiv:1009.5686v1 [hep-ex].

[31] D0 Collaboration, D0 public note 5950-CONF.

[32] CDF Collaboration, CDF public note 9824.

[33] CDF Collaboration, CDF public note 10211.

[34] CDF Collaboration, CDF public note 10224.

[35] D0 Collaboration, D0 public note 6062-CONF.

[36] B. Kilminster, in proceedings of this conference. 